\title{
Classification of rotator cuff tendinopathy using high definition ultrasound
}

\author{
Hannah Hinsley \\ Alex Nicholls \\ Michael Daines \\ Gemma Wallace \\ Nigel Arden \\ Andrew Carr
}

Nuffield Department of Orthopaedics, Rheumatology and Musculoskeletal Sciences, NIHR Biomedical Research Unit, Botnar Research Centre, University of Oxford, UK

Corresponding author:

Hannah Hinsley

Nuffield Department of Orthopaedics, Rheumatology and Musculoskeletal Sciences, NIHR Biomedical Research Unit, Botnar Research Centre, University of Oxford

Nuffield Orthopaedic Centre

Windmill Road

OX3-7LD Oxford, UK

E-mail: hannahoag@doctors.org.uk

\section{Summary}

Background: ultrasound is a valid cost effective tool in screening for rotator cuff pathology with high levels of accuracy in detecting full-thickness tears. To date there is no rotator cuff tendinopathy classification using ultrasound. The aims of this study are to define a valid high-definition ultrasound rotator cuff tendinopathy classification, which has discriminate validity between groups based upon anatomical principles.

Methods: 464 women, aged 65-87, from an established general population cohort underwent bilateral shoulder ultrasound and musculoskeletal assessment. Sonographer accuracy was established in a separate study by comparing ultrasound findings to the gold standard intra-operative findings.

Results: there were 510 normal tendons, 217 abnormal tendons, 77 partial tears, and 124 fullthickness tears. There was no statistical difference in age or the proportion with pain between the abnormal enthesis and partial tear groups, however both groups were statistically older $(p<0.001)$ and had a greater proportion with pain $(p<0.001 \& p=0.050)$ than normal tendons. The full-thickness tears were statistically older than normal tendons $(p<0.001)$, but not abnormal/partially torn tendons. The proportion with pain was significantly greater than both groups $(p<0.001 \&$ $\mathrm{p}=0.006$ ). Symptomatic shoulders had a larger median tear size than asymptomatic shoulders $(p=0.006)$. Using tear size as a predictor of pain likelihood, optimum sensitivity and specificity occurred when dividing tears into groups up to $2.5 \mathrm{~cm}$ and $>2.5 \mathrm{~cm}$, which corresponds with anatomical descriptions of the width of the supraspinatus tendon.

Conclusion: the classification system is as follows: Normal Tendons; Abnormal enthesis/Partial-thickness tear; Single tendon full-thickness tears $(0-2.5 \mathrm{~cm})$; Multi-tendon full-thickness tears $(>2.5 \mathrm{~cm})$.

KEY WORDS: classification, rotator cuff, high-definition ultrasound.

\section{Introduction}

Imaging plays an important role in the diagnosis and management of the painful shoulder. High-definition ultrasound is considered to be a cost effective imaging method for screening of rotator cuff tears. It has been extensively validated, and achieves high levels of accuracy for detecting or ruling out full-thickness tears. From systematic reviews of the literature pooled sensitivities and specificities are 0.95 and 0.96 respectively. Sensitivity figures of 0.98 are reported when using a probe of $10 \mathrm{MHz}$ or greater ${ }^{1,2}$. The major limitation with high definition ultrasound is its failure to accurately detect partial thickness tears. Here sensitivities fall to 0.72 whilst specificities remain high at $0.93^{1}$.

Detection errors and measurement errors have also been examined ${ }^{3,4}$. Misdiagnosis is uncommon with full-thickness tears. However with partial thickness tears it is far more common and the majority of errors are in missed diagnosis. In terms of measurement, errors determined by those estimated outside of $5 \mathrm{~mm}$ from the arthroscopic measurement, are more common when measuring retraction than width of tear. Errors are reported in $25 \%$ of retracted measurements and $12 \%$ of width measurements ${ }^{4}$. Analysis of interobserver variability has also shown to be low when comparing consultant musculoskeletal radiolo- 
gists. Agreement is found in $96 \%$ of cases, and where there was disagreement this was not major ${ }^{5}$. Accuracy levels also depend upon the operator. With the advent of portable high-resolution portable machines, ultrasound has become available to clinicians to use in clinics, and is not restricted to use by musculoskeletal radiologists. However questions have been raised as to the appropriateness of this as ultrasound remains an operator dependent technique. Numerous studies have demonstrated that experienced orthopaedic surgeons have similar high levels of accuracy when diagnosing partial-thickness tears to musculoskeletal radiologists, which is statistically better than general radiologists and sonographers ${ }^{2,6-9}$.

In terms of what equates to 'experienced'; the British Society of Shoulder and Elbow Surgeons (BESS) have produced evidence-based guidelines for best shoulder ultrasound practice by orthopaedic surgeons (www.bess.org.uk). This involves the attendance on a shoulder ultrasound-training course, followed by a brief period of familiarity scanning. Following this a training protocol should be adopted, during which shoulders are scanned pre-operatively on the day of surgery and results compared to arthroscopic findings. This should be reviewed after 50 and 100 scans and predictive values compared to published results. Once achieved the clinical use of ultrasound can be recommended ${ }^{10}$.

Current classification systems: tendons are classified into not torn, partial tears and full-thickness tears. However there is a number of surgeon classifications which attempt to subdivide full-thickness tears into smaller subgroups. These include Cofield (1982) and Bateman (1984) who divided tears into small $(<1 \mathrm{~cm})$, medium (1$3 \mathrm{~cm})$, large $(3-5 \mathrm{~cm})$, and massive $(>5 \mathrm{~cm})$. The Southern California Orthopaedic institute derived a similar classification of small (pinhole), moderate $(<2 \mathrm{~cm}$ including only one tendon with no retraction), large (an entire tendon with retraction), and massive (two or more tendons with retraction). Other systems have evolved around shape (Ellman), retraction extent (Patte) or tear position (Habermayer). There is no validated consensus on how to subdivide full-thickness tears according to size or morphology.

A number of studies have attempted to measure the true anatomical footprint of the supra-spinatus tendon in its average insertion width in the A-P plane. Although there is no absolute consensus most musculoskeletal radiology texts quote an A-P width of 25 $\mathrm{mm}^{11}$. Other studies have been performed on cadavers. Ruotolo used callipers to measure 48 cadaveric specimens with a mean age of 72 years, and reported mean widths of $25 \mathrm{~mm}^{12}$, whilst Roh reported mean widths of $21 \mathrm{~mm}+/-3 \mathrm{~mm}^{13}$. Other studies have commented on the difficulty in making measurements due to the inter-digitation of supra-spinatus and infra-spinatus muscle units and tendon fibres. Minagawa reported that the anterior $12.5 \mathrm{~mm}$ consisted solely of supra-spinatus fibres, and posteriorly another $10 \mathrm{~mm}$ was a combination ${ }^{14}$. Mochizuki reported isolated supra-spinatus footprints to be as small as $16 \mathrm{~mm}$, with the majority of the footprint to be anterior fibres of infra-spinatus sweeping forwards and thus having a combined footprint of $33 \mathrm{~mm}^{15}$.

Arising from our observations in patients, we hypothesised that in tendinopathy and pre-tear abnormalities of the enthesis the normally smooth appearance of the tendon bone interface seen on ultrasound would be replaced with a craggy uneven interface. This appearance should be possible to visualize using ultrasound examination.

The aims of this study are to define a valid classification of rotator cuff tendinopathy using high-definition ultrasound that can be used in the out-patient setting, which has discriminate validity between groups and based upon anatomical and clinical principles. We aim firstly to validate abnormal tendon enthesis as a distinct from normal group as this is easily identified using ultrasound. Secondly we aim to describe a valid sub-categorization of full-thickness tears. Our hypothesis is that the clinical presentation of isolated single tendon tears will be different to that of larger multitendon tears and thus according to the anatomical literature, an ultrasound measurement of a single tendon tear would be up to $25 \mathrm{~mm}$ on the A-P plane.

\section{Patients and methods}

Setting and subjects: the study participants were selected from the Chingford Study, a well-described population-based cohort, derived from the register of a large general practice in Chingford, North London 20 years ago, originally involving 1003 women. The women, currently aged 64-87 years are representative of women in the UK general population with respect to weight, height, and smoking characteristics. With consent and ethical approval a musculoskeletal assessment, and bilateral shoulder ultrasound examination was performed in all of the 463 women who voluntarily attended the 20-year follow up visit.

Pain assessment: pain was defined as the presence of shoulder pain in the previous 4 weeks, and severity defined using the validated numeric rating scale (NRS).

Ultrasound examination protocol: the procedure protocol was fixed and performed by a single operator (343 by Ms HCL Hinsley (Orthopaedic SpR and author), and 123 by Mr A Nichols (Orthopaedic SpR). Data was recorded from the left shoulder then the right on a specific data collection sheet.

The ultrasound examination was performed using the GE volusoni portable ultrasound machine with a 10$16 \mathrm{MHz}$ linear probe. The protocol for ultrasound examination of the shoulder is laid out below, and is based upon the recommendations of the Nuffield Orthopaedic centre musculoskeletal radiology unit protocol. It is performed from standing behind the patient who is seated on a stool to allow easy movement of the arm.

1. Examination of long head of biceps.

The hand is placed on the thigh with the palm facing upwards. The biceps tendon is found in the intertubercular groove of the humerus. Comment is 
made on the presence of the tendon, any fluid surrounding the tendon and the stability of the tendon in the grove when the arm is externally rotated.

2. Examination of subscapularis.

With the arm maximally externally rotated the subscapularis tendon is viewed in the transverse and longitudinal planes from its insertion on the lesser tuberosity to the point at which it becomes hidden behind the coracoid process. The quality of the tendon and the presence/absence of a full thickness tear were noted.

3. Examination of the supraspinatus tendon.

The subject's hand is placed on their back with the palm facing the lumbar spine. This extends and internally rotates the shoulder bringing the tendon out from underneath the acromium where it can now be visualized using ultrasound. The tendon is examined anteriorly in a near sagital plane. The tendon is examined in the longitudinal plane as it inserts into the greater tubersity, and in the transverse plane from the leading edge backwards. Any fluid in the overlying bursa is commented upon, along with the tendon quality and presence of full-thickness tear. If a full thickness tear is present this is measured on the transverse view, and recorded in $\mathrm{cm}$.

4. Examination of the infraspinatus tendon.

By placing the subjects hand on the contralateral shoulder into a flexed and adducted position the infraspinatus tendon is found distal to the spine of the scapula in the longitudinal view. Again the quality and presence of a full- thickness tear are noted.

Tendons were classified into one of 4 working groups, and all tear sizes if present documented. These working groups were created as a result of a consensus meeting, involving the senior author. It was felt that from personal observation that the abnormalities of the tendon enthesis should be included as these represent a clear easily detectable difference on ultrasound to the normal tendon group, and this could potentially provide new evidence for pretear conditions. Partial-thickness tears were included as a separate group as they are clinically managed differently to full-thickness tears.

The groups were as follows (Fig. 1):

Normal tendon

The tendon has a normal homogenous appearance throughout, and where it inserts onto the greater tuberosity there is no abnormal reaction-taking place on the bone side.

\section{Abnormal tendon}

The tendon may appear abnormal with loss of the homogenous appearance or appear abnormally thin. There is an abnormal tendon enthesis, which is clearly visualised as a ragged greater tuberosity. Fluid may be present in the bursa.

\section{Partial-thickness tear}

A lucent patch is visualised in the tendon either on the articular or bursal surface, but there are continu-

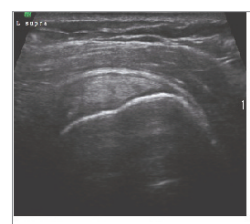

Normal tendon

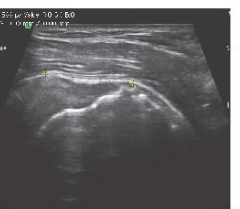

Full-thickness

tear $<2.5 \mathrm{~cm}$

[longitudinal)

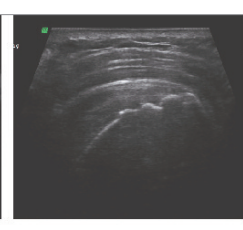

Abnormal tendon

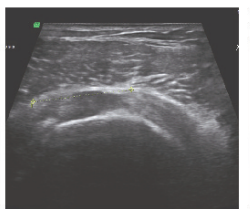

Full-thickness

tear $<2.5 \mathrm{~cm}$

(transverse)

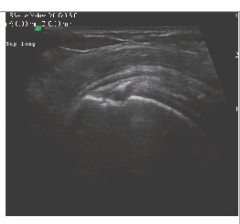

Partial-thickness tear

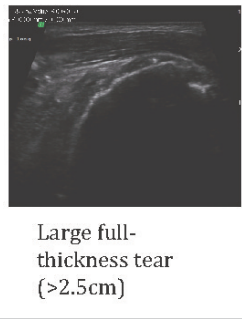

Figure 1. Ultrasound images.

ous tendon fibres inserting into the greater tuberosity. In all cases an abnormal tendon enthesis was observed (even in those with bursal sided tears). Fluid is often seen in the bursa.

\section{Full-thickness tear}

A lucent patch is visualised running through the fullthickness of the tendon. The width of this in the sagital plane is the tear size. This is associated with a concavity in the bursal surface, which is either directly visualised or becomes apparent with pressure from the probe. The tendon enthesis is abnormal and there is fluid in the bursa. In larger tears no tendon is often visible. The surface of the greater tuberosity is abnormal and a heterogenous substance fills the space left by the tendon. In smaller tears a tendon edge may be visible in the longitudinal plane and this measurement is the degree of retraction.

Training and validation: training, followed the protocol laid out in the BESS guidelines (www.bess.org.uk). The initial training took place on the Skills Course in Musculoskeletal Ultrasound held in Oxford in January 2010. Further familiarity experience took place in the outpatient department at the Nuffield Orthopaedic Centre, under the supervision of the senior author, who is well-established in shoulder ultrasound.

Sonogropher validation took place using a local learning curve study ${ }^{10}$. In this study, 50 pre-operative patients awaiting shoulder arthroscopy underwent an ultrasound scan of their shoulder on the morning of their surgery. Participants were blinded to the diagnosis of the patients being scanned. The results were compared to the same day arthroscopy findings.

Two examiners performed the ultrasound examinations in this study; both of whom underwent the same training protocols. Inter-observer validity was performed using statistical comparisons between the distributions of diagnosis after adjusting for age and pain. Intra-observer validity, by repeat scans, was only possible for 18 shoulders due to lack of consulta- 
tion space. Verbal and clinical examinations were not re-performed and no notes from the previous examination were available. The protocol for the ultrasound examination was unchanged.

Statistical analysis: inter-obsever validity was demonstrated comparing the distributions of tendinopathy groups diagnosed on ultrasound after adjustment for age and BMI using the Mann-Witney_U test. Intra-observer validity was demonstrated using a weighted kappa test.

Age, BMI, and pain presence were compared between tendinopathy groups. Age was not normally distributed and BMI was normally distributed. The Mann-Witney-U test, Students T-Test, and Fischer's exact tests were used for non-normal, normal and categorical data respectively. Distributions of tear sizes across symptomatic and asymptomatic shoulders were compared using the Mann-Witney-U test. Correlations between tear sizes and the NRS were demonstrated using a smoothed best-fit curve and spearman's correlation co-efficient. To determine the optimum tear size as a predictor of pain a sensitivity specificity analysis was performed and a receiver operator curve used to predict the best fit model.

All statistics were performed using IBM SPSS Statistics version 22 (IBM, Armonk, NY, USA). The research has been conducted ethically according to international standards as required by the journal as described by Padulo et al. ${ }^{16}$.

\section{Results}

Training and validation results: in the learning curve study ${ }^{10}$ the author's accuracy of diagnosing full-thickness tears was $96.0 \%$ (weighted kappa 0.959 $(p=<0.001))$, with sensitivity $95 \%$, specificity $97 \%$, positive predictive value $95 \%$ and negative predictive value $96.9 \%$. Pearsons correlation coefficient of tear size measurement was $0.761(95 \% \mathrm{Cl} 0.469-0.903$, $\mathrm{p}<0.001)$, and when divided into groups accuracy was $85 \%$. The accuracy of diagnosing partial thickness tears was $88.2 \%$ (weighted kappa 0.557 $(p<0.001))$, with sensitivity $55 \%$, specificity $95.2 \%$, positive predictive value $71.4 \%$, negative predictive value $90.9 \%$. However of the 4 missed partial tears, these were all reported as abnormal footprints.
Intra and inter-observer validity within the general population cohort: there was no significant difference, after adjustment for age and pain, (Mann-Witney- $U$ $p=0.80$ ) in the distributions of diagnosis scanned by each examiner across the cohort. Both examiners scanned two cases and there was $100 \%$ agreement within the classification system. Of the 18 shoulders re-scanned there were 3 disagreements (weighted kappa $0.915 \mathrm{p}<0.001)$. In two cases a previously diagnosed partial-thickness tear was classified as an abnormal tendon. The other disagreement occurred in the size of the full-thickness tear. Here the original diagnosis was a $1.3 \mathrm{~cm}$ full-thickness tear, which was documented as having only a very thin abnormal remnant of tendon visible aside of the tear. Two months later this is recoded as a massive tear with no visible tendon.

Study results: 926 shoulders were included in the study; of which the clinical, demographic and radiological characteristics are shown in Table 1.

Abnormal footprints and partial-thickness tears: there was no significant difference in age, BMI or pain between abnormal tendons and partial thickness tears. However age was significantly different when each group was compared to normal tendons (Mann-Witney $U \mathrm{p}<0.001)$. The proportion with pain was also significantly different between normal tendons and both abnormal tendons (Fishers exact test $p<0.001$ ) and partial-tears (Fishers exact test $p=0.050$ ). Both the abnormal tendons and partial tears had a significantly greater proportion of individuals with pain compared to normal tendons, yet no significant difference between each other (Graph. 1).

Full-thickness tear sizes: 123 full-thickness tears were included in the analysis. There was no difference in BMI between any of the tendinopathy groups. Those with full-thickness tears were significantly older than those with normal tendons (Mann-Witney $U$, $\mathrm{p}<0.001$ ), but not those with abnormal/partial tears. The proportion of full-thickness tears with pain was significantly different from normal (Fishers exact test $\mathrm{p}<0.001$ ), and abnormal/partial tears (Fisher exact test test $p=0.006)$. Tear sizes were not uniformly distributed between symptomatic and asymptomatic

Table 1. Clinical, demographic, and radiological characteristics.

\begin{tabular}{lllll}
\hline & $\mathrm{N}(\%)$ & Median age & Mean BMI & \% Pain \\
\hline Normal & 510 & 71 & 27.5 & 22.8 \\
\hline Abnormal & $(55: 1)$ & & 28.1 & 36.9 \\
\hline Partial tears & 217 & 73 & & 37.7 \\
\hline Full-thickness tears & $77.4)$ & 74 & 27.5 & 51.6 \\
\hline All & $(8.3)$ & 74 & 28.5 & 31.2 \\
\hline
\end{tabular}




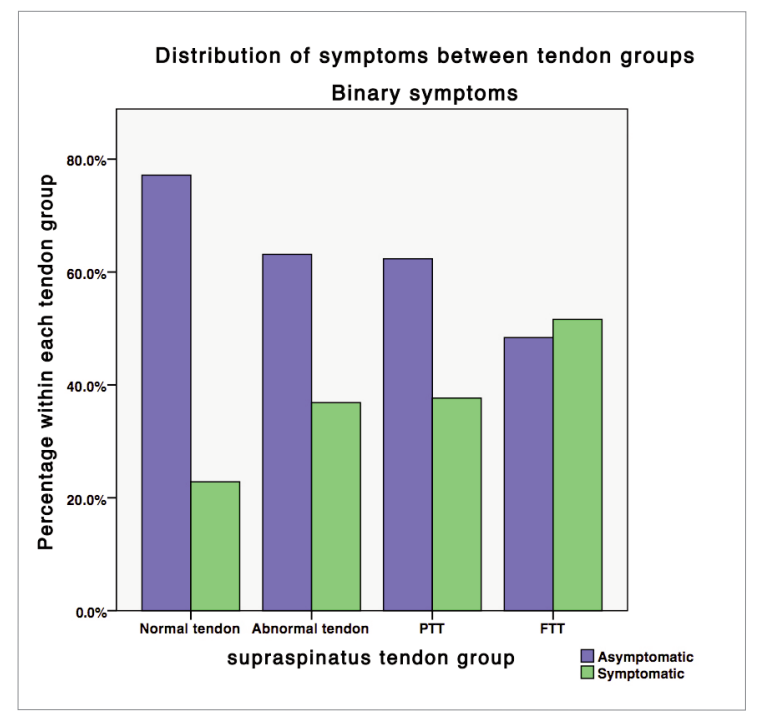

Graphic 1. shoulders, with the median tear size being greater in the symptomatic shoulders (Mann-Witney-U, $\mathrm{p}=0.006$ ) (Graph. 2a).

When correlating the size of tear with the NRS score, the smoothed best-fit line demonstrates a change in pain profile between tear sizes 2 and $3 \mathrm{~cm}$ (spearman correlation co-efficient 0.245, $p=0.006$ ) (Graph. 2b).

For tear size to be predictor of pain, a receiver operating characteristic (ROC) curve was used to define the cut-off values predicting the presence of pain with optimal sensitivity and specificity. This occurred between $0-2.5 \mathrm{~cm}$ and $>2.5 \mathrm{~cm}$.

\section{Discussion}

The overall aim of the study was to produce a valid discriminate classification system of rotator cuff tears that could be applied in the outpatient setting using high-definition ultrasound. Therefore ultrasound has to be able to validly determine the difference between

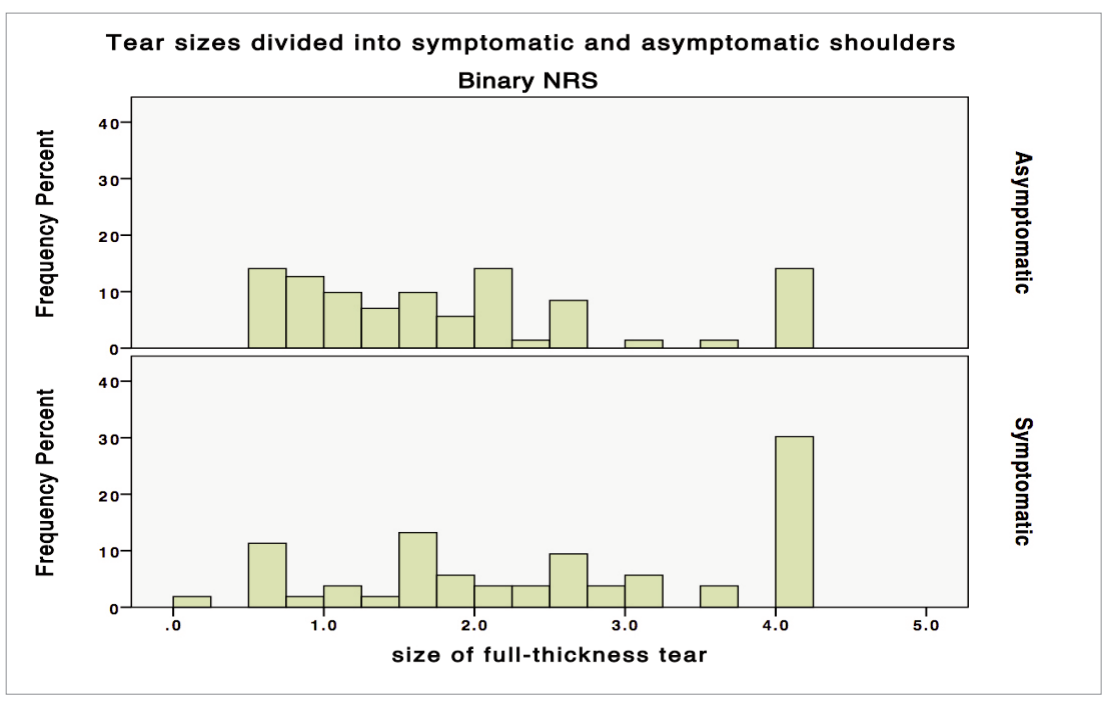

Graphic 2a.

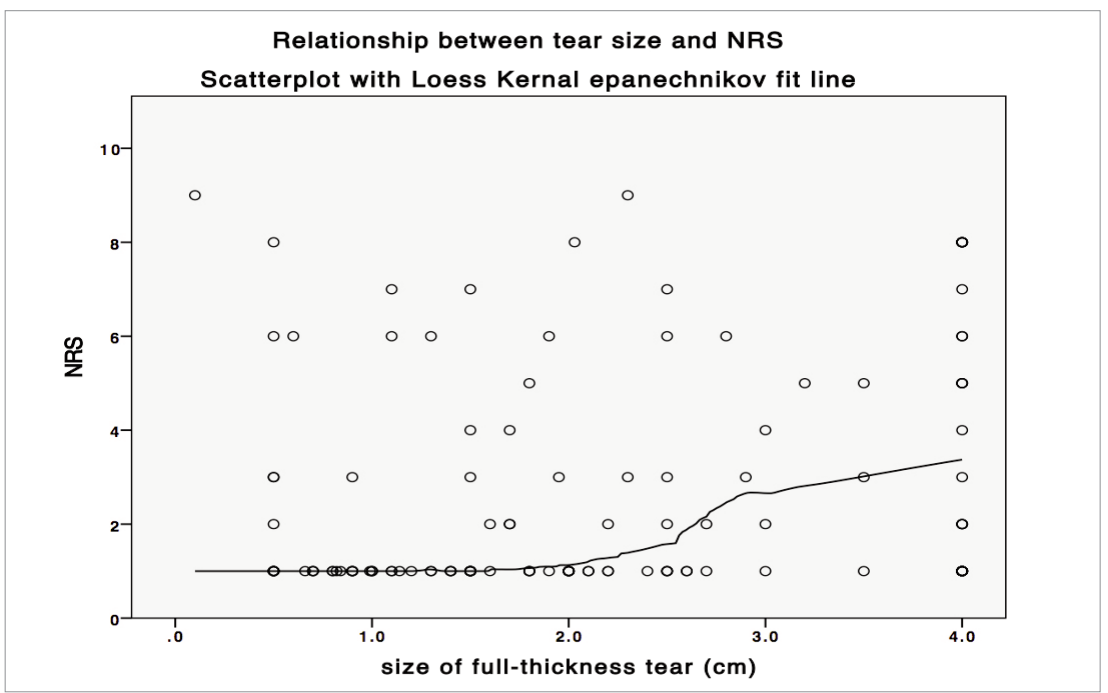

Graphic 2b. 
groups. There were 2 limitations in the study methodology. Firstly, using a single clinician to assess symptoms and perform the ultrasound examination could bias any association between symptoms and pathology, which could have been reduced with two blinded examiners. To minimise this, a strict procedure protocol was followed which had the symptom assessment performed first as this may have been easier to influence. Secondly, a larger intra-observer validity study would have been preferable, but was not possible due to resource constraints.

We have demonstrated firstly that we cannot accurately diagnose partial thickness tears using ultrasound, however can validly identify pre-tear tendinopathy and enthesis pathology. This has been demonstrated by the learning curve study that was undertaken a priory. Here the accuracy rates are comparable with those quoted in the literature, and show that sensitivities for detecting partial thickness tears are significantly lower than that for full-thickness tears where as specificity levels remain significantly higher. This evidence would support any classification system that could distinguish between fullthickness tears vs no full-thickness tears. It would not though, support a separate category for partial thickness tears. From the minimal data that is present regarding the missed partial-thickness tear in the learning curve study, each of these was documented as having an abnormal tendon enthesis. This would support the rationale for having a group in which the tendon is not normal. The personal experience of the author and co-authors is that although partial thickness tears are difficult to detect, this abnormal tendon enthesis is easy to see on ultrasound. Secondly, as shown by the Chingford cohort study, pain proportions and demographic profiles for abnormal tendons and partial tears groups are the same, yet significantly different to normal tendons and full-thickness tears. Thus an abnormal tendon enthesis and partial-thickness tear group combined is a valid and easily identified group which has not previously been described in the literature.

In terms of full-thickness tears we have shown using a general population cohort, that the likelihood of having pain is not uniform across the whole full-thickness tear group, and neither does it change in a linear fashion. We used a sensitivity specificity model to try to determine the point at which full-thickness tears become increasingly likely to be symptomatic, and defined the cut point using the closest to perfect predictive model. This occurred between sizes $0-2.5 \mathrm{~cm}$ and $>2.5 \mathrm{~cm}$. In line with radiological and anatomical studies looking at the supraspinatus footprint this confirmed our hypothesis that the change occurred between single and multi-tendon tears, and likely reflects the altered biomechanics of the shoulder with large multi-tendon tears.

This classification was not designed to sub-categorize individuals into different treatment groups as there are a wide range of treatments available which must be tailored individually. Indeed as this data has come from a general population cohort it does not necessarily demonstrate the self selecting cohort of patients who seek treatment, as in this case $48.4 \%$ of full-thickness tears were pain free. It does however provide a valid tool to clinicians in the outpatient clinic to classify rotator cuff pathology using high-definition ultrasound.

\section{Summary}

Using a general population cohort this study has demonstrated a valid classification of rotator cuff tears using high definition ultrasound that can be applied in the outpatient setting and is based upon anatomical principles and clinical presentations. Each group can validly be identified, and has different profiles in terms of demographics and pain.

The classification system is as follows:

1. Normal Tendons

2. Abnormal enthesis/ Partial-thickness tear

3. Single tendon full-thickness tears $(0-2.5 \mathrm{~cm})$

4. Multi-tendon full-thickness tears $(>2.5 \mathrm{~cm})$.

\section{References}

1. Ottenheijm RP, Jansen MJ, Staal JB, et al. Accuracy of diagnostic ultrasound in patients with suspected subacromial disorders: a systematic review and meta-analysis. Arch Phys Med Rehabil. 2010 ed. 91(10):1616-1625.

2. Smith TO, Back T, Toms AP, Hing CB. Diagnostic accuracy of ultrasound for rotator cuff tears in adults: a systematic review and meta-analysis. Clin Radiol. 2011;66(11):1036-1048.

3. Teefey SA, Hasan SA, Middleton WD, Patel M, Wright RW, Yamaguchi K. Ultrasonography of the rotator cuff. A comparison of ultrasonographic and arthroscopic findings in one hundred consecutive cases. J Bone Joint Surg Am. 2000;82(4): 498-504.

4. Teefey SA, Middleton WD, Payne WT, Yamaguchi K. Detection and measurement of rotator cuff tears with sonography: analysis of diagnostic errors. American Journal of Roentgenology. 2005;184(6):1768-1773.

5. Middleton WD, Teefey SA, Yamaguchi K. Sonography of the rotator cuff: analysis of interobserver variability. American Journal of Roentgenology. 2004;183(5):1465-1468.

6. Iannotti JP, Ciccone J, Buss DD, et al. Accuracy of officebased ultrasonography of the shoulder for the diagnosis of rotator cuff tears. J Bone Joint Surg Am. 2005;87(6):13051311.

7. Moosmayer S, Smith H-J. Diagnostic ultrasound of the shoulder-a method for experts only? Results from an orthopedic surgeon with relative inexpensive compared to operative findings. Acta Orthop. 2005;76(4):503-508.

8. Jeyam M, Funk L, Harris J. Are shoulder surgeons any good at diagnosing rotator cuff tears using ultrasound? A comparative analysis of surgeon vs radiologist. Int J Shoulder Surg. 2008;2(1):4-6.

9. Al-Shawi AK, Badge R, Bunker TD. Reliability of shoulder ultrasound to detect rotator cuff tears when performed by the surgeon in the place of first contact. Journal of Bone. 2012.

10. Murphy RJ, Daines MT, Carr AJ, Rees JL. An independent learning method for orthopaedic surgeons performing shoulder ultrasound to identify full-thickness tears of the rotator cuff. J Bone Joint Surg Am. 2013;95(3):266-272.

11. O'Neill JMD. Musculoskeletal Ultrasound. Springer. 2008. 1 p.

12. Ruotolo C, Fow JE, Nottage WM. The supraspinatus footprint: 
an anatomic study of the supraspinatus insertion. Arthroscopy 2004;20(3):246-249.

13. Roh MS, Wang VM, April EW, Pollock RG, Bigliani LU, Flatow EL. Anterior and posterior musculotendinous anatomy of the supraspinatus. J Shoulder Elbow Surg. 2000;9(5):436-440.

14. Minagawa $\mathrm{H}$, Itoi $\mathrm{E}, \mathrm{Konno} \mathrm{N}$, et al. Humeral attachment of the supraspinatus and infraspinatus tendons: an anatomic study. Arthroscopy. 1998;14(3):302-306.
15. Mochizuki T, Sugaya H, Uomizu M, et al. Humeral insertion of the supraspinatus and infraspinatus. New anatomical findings regarding the footprint of the rotator cuff. Surgical technique. J Bone Joint Surg Am. 2009;91Suppl 2 Pt 1:1-7.

16. Padulo J, Oliva F, Frizziero A, Maffulli N. Muscles, Ligaments and Tendons Journal. Basic principles and recommendations in clinical and field science research. MLTJ. 2013;4: 250-252. 